\title{
EDITORIAL
}

\section{Editorial zum Schwerpunktthema: Universitätsschulen}

\section{Editorial to the Focus Topic: University Schools}

Die bildungspolitisch in vielen Bundesländern deutlich gestärkte Praxisorientierung, z.B. durch die Einführung von Praxissemestern und verlängerten Praxisphasen in der Lehrerbildung, führt zu einer systematischeren Vernetzung von lehrerbildenden Hochschulen mit Schulen. Damit sind zuweilen nicht nur die intensivierte Kooperation im Rahmen von Praktika oder insgesamt der Ausbau der kooperativen Praxisphasen in der Lehrerbildung, sondern auch eine dezidierte Forschungsabsicht der Universitäten verbunden.

Im Vergleich zeigt sich, dass die Kooperationskonzepte sich nicht nur durch unterschiedliche Grade der Institutionalisierung auszeichnen, sondern auch Akzentsetzungen aufweisen, die einerseits in Richtung einer stärkeren Forschungsorientierung und andererseits einer starken Ausbildungsorientierung gehen. Hiermit setzt in Deutschland eine Ausdifferenzierung der institutionalisierten Kooperationsformen von Universitäten und Schulen ein, die im Heft im Hinblick auf „Universitätsschulen“ als Kooperationsprojekte mit besonders hohem Institutionalisierungsgrad dargestellt wird.

Die an den jeweiligen Universitätsschulen fällige Strukturentscheidung für das Konzept der Ausbildungs- und/oder Forschungsschule lässt sich auch schon in der Geschichte der Kooperation von Schulen und Universitäten nachzeichnen. Ausgehend von einer bildungshistorischen Perspektivierung des Phänomens werden zum Vergleich, zum Abgleich und zur Horizonterweiterung sowohl internationale Beispiele aus den USA, England und Österreich als auch traditionsreiche und neu entstehende innovative Kooperationsformen aus Deutschland dargestellt.

In dem den Schwerpunkt aus historischer Perspektive einleitenden Beitrag zeigt Eva Matthes, dass das Phänomen der Universitätsschule eine bedeutsame Tradition hat, wie die Autorin anhand von mehreren Beispielen aus der Geschichte der Schulforschung bzw. universitären Lehrerbildung zeigt. Der Beitrag lässt erkennen, welche Alternativen es neben derzeit aktuellen, im Heft dokumentierten Konzepten gegeben hat und welche der damaligen pädagogisch-systematischen Erwägungen auch 
heute noch bedenkenswert sind. Als das wahrscheinlich prominenteste Beispiel innerhalb Deutschlands mag hier die Jena-Plan-Schule von Peter Petersen gelten, die zunächst als Übungsschule, später als Universitätsschule bis zum Jahr 1950 existierte.

In Deutschland blieben institutionalisierte Kooperationen von Universität und Schule jedoch lange Zeit die Ausnahme, ganz anders als in anderen Bildungssystemen, wo universitäre Praxisschulen bzw. sogenannte Campus-Schulen vielfach über eine lange Tradition verfügen, dabei allerdings nicht immer kontinuierliche Entwicklungslinien aufweisen.

So wirft der zweite Schwerpunktbeitrag einen Blick nach England. Chris Brown reflektiert die grundsätzlichen Herausforderungen und Möglichkeiten von „school/ university partnerships“ zwischen „research“ und „development“ und arbeitet relevante Bedingungen gelingender Kooperationen heraus, etwa im Hinblick auf geteilte Ziele der beteiligten Akteure, die Rolle der Kooperationskultur und die Relevanz strategisch denkender Führungspersonen. Auch die Bedeutung eines „third space“ für mehr kreative Arbeitsweisen jenseits der jeweiligen Institutionslogiken und Organisationskulturen wird thematisiert.

In Österreich gehören sogenannte „Übungshauptschulen“ seit langem sogar zum festen Bestandteil der akademischen Lehrkräfteausbildung, wie Marlies Krainz-Dürr in ihrem Beitrag zeigt. Bemerkenswert ist hier, dass die Übungsschulen sich zunehmend auch dem Forschungs- und Entwicklungsparadigma annähern.

In den USA gibt es ebenfalls eine lange Tradition sogenannter „university-affiliated schools“, wie Frank C. Worrell von der University of California, Berkeley, nachzeichnet. Vom 18. Jahrhundert bis heute gab es drei Wellen einer entsprechenden Bewegung, die von unterschiedlichen Motiven und Zielen geprägt waren. Die UC Berkeley kann ebenfalls auf eine lange institutionalisierte Kooperation mit einer Schule zurückblicken. Worrell zeichnet in seinem Beitrag die Entstehungsgeschichte und die Rolle etablierter Strukturen und klarer Verantwortlichkeiten für eine dauerhafte und nachhaltige Kooperation nach. Dabei nimmt er auch die aus US-amerikanischer Perspektive wichtige Frage der Rolle von Stiftungen und externer Finanzierung in den Blick.

Als noch aus der letzten großen Reformphase in Deutschland existierende Institutionen erproben die bereits 1974 am Universitätsstandort Bielefeld gegründeten Versuchsschulen des Landes Nordrhein-Westfalen - „Laborschule“ und „OberstufenKolleg“ - mit ihrem Lehrerforschermodell exemplarische Formen des Zusammenspiels von Forschung und Entwicklung. So existieren, wie Monika Palowski, Johanna Gold und Gabriele Klewin zeigen, an den Bielefelder Versuchsschulen ein staatlicher Versuchsauftrag mit langer Tradition und einem fest verankerten Selbstverständnis der Lehrenden als Forschende sowie eine kontinuierliche Kooperation mit Wissen- 
schaftler*innen, deren Arbeitsschwerpunkt die gemeinsame Forschung und Entwicklung im Sinne der Schul- und Unterrichtsentwicklung von Reformschulen ist.

Demgegenüber verweist Kersten Reich auf eine anderweitige Akzentsetzung, wenn die forschungsorientierte Verbesserung der Lehrer(aus)bildung im Mittelpunkt steht, für die die Inklusive Universitätsschule Köln (IUS Köln) mit dem Konzept einer universitären Praxisschule zeichnet.

Zwar existieren auch in Hessen Versuchsschulen des Landes, am prominentesten hier wohl die Helene-Lange-Schule Wiesbaden, deren wissenschaftliche Begleitung und Erforschung durch die Universität aber nicht vergleichbar fest institutionalisiert ist wie an der Universität Bielefeld oder der Universität Köln und immer wieder neu ausgehandelt werden muss, wie Barbara Asbrand und Carmen Bietz in ihrem Beitrag zeigen.

Insgesamt zeichnen sich für das zunehmend praxisorientierter angelegte und stetig weniger vom Schisma der ersten und zweiten Phase der Lehrerbildung geprägte Lehramtsstudium hierzulande Veränderungen ab. Mit dem Blick ins Ausland und auf internationale Konzepte der Lehrerausbildung werden zunehmend praxisorientierte alternative Kooperationen von Universitäten und Schulen diskutiert. Aber auch (Ausbildungs-)Konzepte mit einer dezidierten Forschungsabsicht der Universitäten im Sinne einer forschungsorientierten Kooperation sind zu finden, wie sich dies im abschließenden Beitrag zum Themenschwerpunkt exemplarisch an den Kooperationsschulen der Universität Koblenz-Landau ablesen lässt, die Alexander Kauertz, Stefanie Würtz und Regina Schleicher in den Blick nehmen.

Martin Heinrich \& Isabell van Ackeren

https://doi.org/10.31244/dds.2019.01.01 